\title{
Prevalence of gyrA and parE mutations in clinical isolates of Streptococcus pneumoniae with decreased susceptibilities to different Fluoroquinolones
}

\author{
Laila M. Yousif ${ }^{1}$, Ghada A. Ismael ${ }^{2}$, Ashraf k. Mohammed ${ }^{3}$ and \\ Department of Clinical Pathology, Faculty of Medicine, Sohag University ${ }^{1,3,4}$ \\ \&Ain Shams University ${ }^{2}$
}

\begin{abstract}
Introduction: Streptococcus pneumoniae is a major Gram-positive pathogen responsible for pneumonia, bactermia, otitis media, and meningitis leading to considerable morbidity and mortality among children and elderly individuals. The primary goals of antibiotic treatment of respiratory tract infections are clinical efficacy of treatment, pathogen eradication, and prevention of resistance development. Resistance to fluoroquinolones in S. pneumoniae arises in a stepwise fashion and results from alterations in the target binding site due to the acquisition of spontaneous mutations in the quinolone resistance-determining regions (QRDRs) of the topoisomerase IV and DNA gyrase genes. Although mutations usually occur in the QRDRs of parC and gyrA, a role for mutations in the parE subunit in low-level resistance has been reported.

Aim of the work: The aim of this study was to determine the prevalence of fluoroquinolone resistance Streptococcus pneumoniae (FQRSP) and to examine the genetic relatedness of pneumococcal isolates with parE and gyrA genes mutations in different specimens in Sohag University Hospital.

Patients and Methods: This study was prospectively conducted over a period of 24 months between October 2015 and September 2017, at Sohag university hospital. During the study period, 78 patients hospitalized for a syndrome consistent with a diagnosis of community acquired pneumonia (CAP ) included in this study with a mean age of 34.5 years (range, 2 to 67 ), $60 \%$ of whom were males. A CAP syndrome was defined as a newly recognized pulmonary infiltrate together with 2 of the following findings: subjective fever or documented temperature $37.4{ }^{\circ} \mathrm{C}$, increased cough, sputum production, or shortness of breath, pleuritic chest pain, confusion, rales, leukocytosis, (according to age) ${ }^{(1)}$. Patients who had taken antibiotic treatment within 3 days prior to initial visit were excluded from this study.

Results: Our study illustrate the role of mutation in the gyrA\&parE genes and the effect of mutations in the both genes in fluoroquinolone resistance among $\mathrm{S}$. pneumoniae isolates.

Conclusion: The present study provide an opportunity to view the predominant mutations conferring reduced susceptibility to FQs in clinical pneumococcal isolates. There is a strong relationship between these mutations and decrese susceptibility to the most fameous FQs to some extent, although this varies between strains and for each drug.
\end{abstract}

Key words: GyrA, ParE, Streptococcus pneumonia, Fluoroquinolones.

\section{Introduction}

Streptococcus pneumoniae is a major Gram-positive pathogen responsible for 
pneumonia, bactermia, otitis media, and meningitis leading to considerable morbidity and mortality among children and elderly individuals. The primary goals of antibiotic treatment of respiratory tract infections are clinical efficacy of treatment, pathogen eradication, and prevention ofresistance development. Penicillin, a $\beta$-lactam antibiotic, has long been the mainstay against pneumococcal infections, but the worldwide spread of antibioticresistant clones over the past decades has impaired its usefulness for dealing with S. pneumoniae infections. The rates of resistance against $\beta$-lactams and macrolides among $S$. pneumoniae isolates have translated into anincreased usage of fluoroquinolone antibiotics in the treatment of respiratory diseases (2). Since their introductionintoclinical use the fluoroquinolones havehada major impact on the treatment of moderateto-severe infections. Their broad spectrum ofactivity,clinical utility, availability in bothoraland parenteralforms, andfavorable

pharmacokinetic

propertieshascontributed

theirextensiveworldwideuse.

However, in recentyearsbacterial resistance

thefluoroquinoloneshasbecome a majorconcern. Fluoroquinolones are part of aclassof synthetic broadspectrumantibioticsthat inhibit DNA synthesis inbacteriaby targeting DNA gyrase (GyrAand-B subunits) and topoisomeraseIV(ParCand -E subunits), two enzymesthatare vital for DNA supercoiling and chromosome segregation,respectively ${ }^{(3)}$.

The rise ingram-positivepathogen resistance in recent yearshasprompted the pharmaceutical industrytodevelop fluoroquinolones withgreateractivity against these rapidlychangingpathogens.Structural modifications

tothebasicfluoroquinolone nucleus havegivenriseto several new generations of compounds. With eachnewgeneration the potency againstmanygram-positive pathogens, including S.pneumoniae,hasimproved.Although the worldwide prevalence offluoroquinolone-resistantS.

pneumoniae remains low in relation to $\beta$-lactam resistance $(<1 \%)$, the dissemination of successful resistant clones has nonetheless increased the prevalence in some countries (4).

Resistance to fluoroquinolones in S. pneumoniae arises in a stepwise fashion and results from alterations in the target binding site due to the acquisition of spontaneous mutations in the quinolone resistance-determining regions (QRDRs) of the topoisomerase IV and DNA gyrase genes. Although mutations usually occur in the QRDRs of parE and gyrA, a role for mutations in the parE and gyrAsubunits in low-level resistance has been reported ${ }^{(5)}$.

Inappropriate use of any antibiotic can contribute to the emergence of resistance to that and related agents. So much work is needed to identify optimal strategies to prevent the emergence and spread of resistant pneumococcal strains in long-term care facilities, including potential use of pneumococcal conjugate vaccines, antimicrobial stewardship, and infection control interventions to interrupt transmission (6).

\section{Aim of the work}

The aim of this study was to determine the prevalence of fluoroquinolone resistance Streptococcus pneumoniae (FQRSP) and to examine the genetic relatedness of pneumococcal isolates with parE and gyrA genes mutations in different specimens.

Patients and Methods: 
Patients: This study was prospectively conducted over a period of 24 months between October 2015 and September 2017, at Sohag university hospital. During the study period, 78 patients hospitalized for a syndrome consistent with a diagnosis of CAP included in this study with a mean age of 34.5 years (range, 2 to 67 ), $60 \%$ of whom were males.

A CAP syndrome was defined as a newly recognized pulmonary infiltrate together with 2 of the following findings: subjective fever or documented temperature $37.4{ }^{\circ} \mathrm{C}$, increased cough, sputum production, or shortness of breath, pleuritic chest pain, confusion, rales, leukocytosis, (according to age) (1). Patients who had taken antibiotic treatment within 3 days prior to initial visit were excluded from thisstudy.

\section{Methods}

\section{Specific investigations}

Clinical specimens and clinical laboratory work.

Fresh sputum samples were collected soon after collection of data from patients (75 specimens). Representative sputum originating from the lower respiratory tract was defined as that containing $>25$ granulocytes and $<10$ epithelial cells per low power field (lpf: total magnification: $\times 100)^{(\mathbf{1})}$.

Bronchoalveolar lavage (BAL) (3 specimens) as diagnostic techniques were used according to the clinical judgment of the physician in charge for some neonates.

\section{Microbiologic Evaluation Culture method}

Isolates were incubated in plates with increased $\mathrm{CO} 2(5-10 \%)$ in order to enhance the development of hemolytic zones of the pathogenic Streptococci and incubated for 18-24 hours. In all cases, growth requires a source of catalase (e.g. blood) to neutralize the large amount of $\mathrm{H} 2 \mathrm{O} 2$ produced by the bacteria.

By gram stain isolates appear as lancetshaped, Gram-positive diplococci or chains of cocci.

The identification of bacteria in our samples was completed by the VITEK ${ }^{\circledR} 2$ Compact System. As a commercialand standard system, its accuracy has been strictly evaluated.

\section{Susceptibility product information Intended Use}

The VITEK ${ }^{\circledR} \quad 2$ Antimicrobial Susceptibility Tests (AST) are intended for use with the VITEK ${ }^{\circledR} 2$ Systems for the automated quantitative or qualitative susceptibility testing of isolated colonies for most clinically significant aerobic Gram-negative bacilli, Staphylococcus spp., Enterococcus spp., Streptococcus spp., S. pneumoniae, and yeast.

\section{Summary and Explanation of the Test}

Susceptibility testing is indicated for any organism that contributes to an infectious processwarranting antimicrobial

chemotherapy. Susceptibility tests are most often indicated when the causative organism is thought to belong to a species capable of exhibiting resistance to commonly used agents. Isolated colonies of each type of organism that may play a pathogenic role are selected from an agar plate and tested for susceptibility. These tests are then examined and the Minimum Inhibitory Concentration (MIC) is determined. The MIC obtained using a dilution test may tell the physician the concentration of an antimicrobial agent needed at the site of infection to inhibit the infecting organism. MICs have traditionally been determined using antimicrobial concentrations derived from Serial twofold dilutions of MIC is then determined from the lowest 
concentration that exhibits inhibition of growth. An interpretive criterion (Susceptible, Intermediate, or Resistant) can then be assigned to MIC results to aid in the direction of therapy. For some antimicrobials (e.g., high-level gentamicin, high-level streptomycin) a qualitative result is generated. The standard and reference procedures are based on susceptibility tests requiring 16 to 24 hours of incubation for bacteria and 24 to 48 hours for yeast. Various manufacturers have now developed automated procedures designed togenerateresults more rapidly by using shortenedrim. Then the DNA was centrifuged at incubation times. Laboratories worldwide14000 rpm for 30 seconds. This wash use either variations of the standardstepwasrepeated.

reference procedure or a commercially available product to determine the MICs of

$\geq 25$ ul DNA Elution Buffer wasinfectious organisms.

Polymerase chain reaction(PCR):-

Simple PCR was performed for all strains to detectfluoroquinolone resistance Streptococcus pneumoniae (FQRSP) and to examine the genetic relatedness of pneumococcal isolates with parE and gyrA genes mutations.

\section{i) Bacterial DNApurification.}

By the use of DNA Clean\& Concentrator

TM-25 (catalog No D4033).

Before starting: $26 \mathrm{ml} \mathrm{95 \%} \mathrm{ethanol} \mathrm{was}$ added to the $6 \mathrm{ml}$ DNA Wash Buffer concentrate.

Protocol:- (according to manufacture instruction)

1- The DNA bands were exiced from the agarose gel with a sterile razor plate and placed in a $1.5 \mathrm{ml}$ microcentrifuge tube, 5 volumes of DNA Binding Buffer were added to each volume of DNA samples placed at $50{ }^{\circ} \mathrm{C}$ for 10 minutes. Then mixed by pulse- vortexing for 15 seconds every 2-3 minutes till the agarose completely dissolved.

2- Then the DNA (including the precipitate) was carefully added to a provided Zymo-Spin ${ }^{\mathrm{TM}}$ Column in a 2 $\mathrm{ml}$ Collection Tube without wetting the rim. Then the cap was closed to avoid aerosol formation duringcentrifugation.

3- The DNA was centrifuged at 14000 rpm for 30 seconds. Then the ZymoSpin ${ }^{\mathrm{TM}}$ Column was placed in a clean $2 \mathrm{ml}$ collection tube and the tube contating the filterate wasdiscarded.

4200 ul DNA Wash Buffer was added to the column without wettingthe added directly to the column matrix without wetting the rim and incubate at room temperature for one minute. The Zymo-Spin ${ }^{\mathrm{TM}}$ Column was placed in a clean $1.5 \mathrm{ml}$ microcentrifuge tube then centrifuged at $14000 \mathrm{rpm}$ for 30 seconds to elute theDNA.

Ultra-Pure DNA was ready to use for the next sequencing step.

\section{ii) DNAsequencing}

Sequencing reactio were prepared with ABI Prism ${ }^{\circledR}$ BigDye Terminator Cycle Sequencing Ready Reaction Kit using conditions descriped by Zhanel et al. (8) with ABI 377 automated sequencer (PE Applied Biosystems, mississauga, ON).

\section{Sequence analysis}

DNA sequences were analyzed with DNASIS 2.6 Sequence Analysis Programs (Hitachi Software Engineering Co., Ltd., San Francisco, Calif.) against 1 of the 2 identical sequenced pneumococcal strains in the database (NC_008533 Streptococcus pneumoniae D39 and AE007317). D39 is a

historically important serotype 2 strain that was used in experiments 
by Avery and coworkers to material.

demonstrate that DNA is the genetic

\section{Results}

During the period from October 2015 and September 2017 our study was carried out in the Clinical Pathology Department, faculty of medicine, Sohag University Hospital, 78 participants included in our study, the majority of the isolates were isolated from males, $49(62.8 \%)$ male and $29(37.2 \%)$ female, aged ranged from 1 to 64 years with mean age $22.2 \pm 20.8$. Inflammatory biomarkers differentiating viral from bacterial infections have been evaluated in our study to support clinical diagnosis. The majority of our study group had high ESR values 65 (83.3\%), and positive CRP 64 (82.1\%). Also in our study, it was observed that WBCs was high in $63 / 78(82.1 \%)$ of cases. S. pneumoniae was isolated from 78 patients included in this study. The resistance percentages of all strains to tested antibiotics were as follows: $91 \%$ of isolates in our study were resistant to Ampicillin, $5.1 \%$ were intermediate and $3.8 \%$ were susceptible. Regarding Cefaclor $83.3 \%$ were resistant, $7.7 \%$ were intermediate and $9 \%$ were susceptible. Erythromycin was resistant in $82.1 \%$ of isolates, intermediate in $10.3 \%$, and susceptible in $7.7 \%$. Regarding Imipenem $10.3 \%$ of isolates were resistant. Tetracycline was resistant in $71.8 \%$ of isolates. Clarithromycin was resistant in only $6.4 \%$, also $10.3 \%$ of our isolates were resistant to ceftriaxone. Trimethoprim/ sulfamethoxazole was resistant in $9 \%$ of ourisolates.

Break points of antibiotics "Ampicillin" $\geq 2 \mathrm{R} \& 0.12-1 \mathrm{I} \& \leq 0.06 \mathrm{~S}$, "Cefaclor" $>16 \mathrm{R} \& 8-16 \mathrm{I} \& \leq 4 \mathrm{~S}$, "Erythromycin" $\geq 1 \mathrm{R} \& 0.5 \mathrm{I} \& \leq 0.25 \mathrm{~S}$, "Imipenem" $\geq 1$ $\mathrm{R} \& 0.5 \mathrm{I} \& \leq 0.25 \mathrm{~S}$ "Tetracyclin" $\geq 8 \mathrm{R} \& 4 \mathrm{I} \& \leq 2 \mathrm{~S}$, "Clarithromycin" $\geq 2 \mathrm{R} \& 1 \mathrm{I}$ $\& \leq 0.5 \mathrm{~S}$, "Ceftriaxone" $\geq 2 \mathrm{R} \& 1 \mathrm{I} \& \leq 0.5 \mathrm{~S}$ and "Trimethoprim-Sulfamethaxzole " $\geq 4$ R \& $1-2$ I \& $\leq 0.5 S$.

Among 78 isolates $37(47.4 \%)$ of S. pneumonia isolates were Fluroquinolones susceptible $12(15.4 \%)$ were with variable susceptibility and 29 (37.2\%) were Fluroquinolones resistant.

The MICs of Ciprofloxacin, Levofloxacin, Gatifloxacin and Moxifloxacin were measured and results were as follow, $44.9 \%$ of S. pneumonia isolates were resistant to ciprofloxacin, $11.5 \%$ were intermediate and $43.6 \%$ were sensitive. Regarding levofloxacin $42.3 \%$ of isolates were resistant, $9 \%$ were intermediate, and $48.7 \%$ were sensitive. Over forty six (46.1\%) of our isolates were resistant to Gatfloxacin, $10.3 \%$ were intermediate, and $43.6 \%$ were sensitive. Regarding Moxifloxacin $46.2 \%$ of our isolates were resistant, $7.6 \%$ were intermediate, and $46.2 \%$ were sensitive (Table 1). Break points of fluroquinolones group "Ciprofloxacin" $\geq 4 \mathrm{R} \& 2 \mathrm{I} \& \leq 1 \mathrm{~S}$ and "Levofloxacin" $\geq 8 \mathrm{R} \& 4 \mathrm{I} \& \leq 2 \mathrm{~S}$ and "Gatfloxacin" $\geq 4 \mathrm{R} \& 2 \mathrm{I} \& \leq 1 \mathrm{~S}$ "Moxifloxacin" $\geq 4$ R \& 2 I $\& \leq 1 \mathrm{~S}$. 
SOHAG MEDICAL JOURNAL of Streptococcus

Vol. 22 No.1 Jan 2018
Prevalence of gyrA and parE mutations in clinical isolates

Mohammed H. Mahmoud

Table (1) Fluroquinolones susceptibility of S. pneumoniae.

\begin{tabular}{|c|c|c|}
\hline Variable & MIC(ug/dl) & no $(\%)$ \\
\hline & Resistant $\geq 4$ & $35(44.9 \%)$ \\
\hline \multirow{2}{*}{$\begin{array}{l}\text { Ciprofloxacin } \\
\left(1^{\text {st }} \text { generation Fluoroquinolone) }\right.\end{array}$} & Intermediate 2 & $9(11.5 \%)$ \\
\hline & Susceptible $\leq 1$ & $34(43.6 \%)$ \\
\hline \multirow{3}{*}{$\begin{array}{l}\text { Levofloxacin } \\
\left(2^{\text {nd }} \text { generation Fluoroquinolone }\right)\end{array}$} & Intermediate4 & $7(9 \%)$ \\
\hline & Susceptible $\leq 2$ & $38(48.7 \%)$ \\
\hline & Resistant $\geq 4$ & $36(46.1 \%)$ \\
\hline \multirow{3}{*}{$\begin{array}{l}\text { Gatfloxacin } \\
\left(3^{\text {rd }} \text { generation Fluoroquinolone }\right)\end{array}$} & Intermediate2 & $8(10.3 \%)$ \\
\hline & Susceptible $\leq 1$ & $34(43.6 \%)$ \\
\hline & Resistant $\geq 4$ & $36(46.2 \%)$ \\
\hline \multirow{2}{*}{$\begin{array}{l}\text { Moxifloxacin } \\
\text { (4 }{ }^{\text {th }} \text { generation Fluoroquinolone) }\end{array}$} & Intermediate 2 & $6(7.6 \%)$ \\
\hline & Susceptible $\leq 1$ & $36(46.2 \%)$ \\
\hline
\end{tabular}

\section{Sequencing of the Ouinolone Resistance-}

\section{Determining(ORDRs).}

Of the 41 quinolone resistant and intermediate isolates isolates, $9(22.0 \%)$ had no substitutions in the QRDRs of either GyrA or ParE, 19 (46.3\%) had a QRDR GyrA substitution, while 16 (39.0\%) had QRDRs substitutions in ParE.

The specific substitutions observed in in GyrAwere Ser81Phe, Ser81Tyr and Glu85Lys. The specific substitutions observed in ParEwere Asp435Asn and Glu407Lys. The percent of isolates with each of the aforementioned substitutions is presented in table (2).

Table (2) Percent and types of the 41 Fluroquinolones-resistant S. pneumoniae isolates with resistance-associated QRDR substitutions.

\begin{tabular}{|l|l|l|}
\hline Variable & & no (\%) \\
\hline & Glu85Lys & $1(2.4 \%)$ \\
\hline GyrA & Ser81Phe & $13(31.7 \%)$ \\
\hline & Ser81Tyr & $5(12.2 \%)$ \\
\hline ParE & Asp435Asn & $6(14.6 \%)$ \\
\hline & Glu407Lys & $2(4.9 \%)$ \\
\hline
\end{tabular}

Table 3 show percentage of genes mutations in each antibiotic of Fluroquinolones-resistant S. pneumoniae

\begin{tabular}{|l|c|c|}
\hline Variable & Gyr A & Par E \\
\hline Ciprofloxacin & $\mathbf{1 8 ( 5 6 . 3 \% )}$ & $\mathbf{8 ( 2 5 \% )}$ \\
\hline Levofloxacin & $18(56.3 \%)$ & $\mathbf{8 ( 2 5 \% )}$ \\
\hline Gatfloxacin & $18(56.3 \%)$ & $\mathbf{8 ( 2 5 \% )}$ \\
\hline Moxifloxacin & $\mathbf{1 8 ( 5 6 . 3 \% )}$ & $\mathbf{8 ( 2 5 \% )}$ \\
\hline
\end{tabular}

As shown in table 4, at Ciprofloxacin MIC 2, 4, 8 and 16, 75\%, 55.6\%, 33.3\% and 30\% had no substitution in $\mathbf{G y r} \mathbf{A}$, only $10 \%$ of ciprofloxacin resistantand intermediate isolates had Glu85Lys substitution at MIC 16. Also at MIC 2, 4, 8 and 16, there were $25 \%, 33.1 \%, 66.7 \%$ and $30 \%$ Ser81Phe substitution respectively. At MIC 4 and 16 there were $11.1 \%$ and 30\% had Ser81Tyr substitution. RegardingParE,atCiprofloxacinMIC2,4,8and16,50\%,66.7\%,77.85and $10 \%$ hadnosubstitutionatParE. $25 \%, 22.2 \%, 22.2 \%$ and $10 \%$ hadAsp 435 Asn substitution. Only $25 \%$ and $11.1 \%$ at MIC 1 and 4 had Glu407Lys substitution. 
SOHAG MEDICAL JOURNAL of Streptococcus

Vol. 22 No.1 Jan 2018
Prevalence of gyrA and parE mutations in clinical isolates

Mohammed H. Mahmoud

Table (4) Ciprofloxacin MICs and substitutions observed in GyrA and ParE in ciprofloxacinresistant and intermediate $\mathrm{S}$. pneumoniae isolates.

\begin{tabular}{|c|c|c|c|c|}
\hline Variable(MIC) & 2 & 4 & 8 & 16 \\
\hline \multicolumn{5}{|l|}{ GyrA } \\
\hline No substitution & $3(75 \%)$ & $5(55.6 \%)$ & $3(33.3 \%)$ & $3(30 \%)$ \\
\hline Glu85Lys & $0(0 \%)$ & 0(0\%) & O (0\%) & $1(10 \%)$ \\
\hline Ser81Phe & $1(25 \%)$ & $3(33.1 \%)$ & $6(66.7 \%)$ & $3(30 \%)$ \\
\hline Ser81Tyr & $0(0 \%)$ & $1(11.1 \%)$ & $0(0 \%)$ & $3(30 \%)$ \\
\hline \multicolumn{5}{|l|}{$\operatorname{Par} \mathbf{E}$} \\
\hline No substitution & $2(50 \%)$ & $6(66.7 \%)$ & $7(77.8 \%)$ & $9(90 \%)$ \\
\hline Asp435Asn & $1(25 \%)$ & $2(22.2 \%)$ & $2(22.2 \%)$ & $1(10 \%)$ \\
\hline Glu407Lys & $1(25 \%)$ & $1(11.1 \%)$ & 0(0\%) & $0(0 \%)$ \\
\hline
\end{tabular}

As shown in table (5) at Levofloxacin MIC 4, 8 and 16, 100\%, 53.8\% and $21.4 \%$ had no substitution in Gyr A, only 7.7\% of levofloxacin resistant and intermediate isolates had Glu85Lys substitution at MIC 8. Also at MIC 8 and 16, there were 30.8\% and 57.2\% Ser81Phe substitution respectively. At MIC 8 and 16 there were $7.7 \%$ and $21.4 \%$ had Ser81Tyrsubstitution.

Regarding Par E, at Levofloxacin MIC 4, 8 and 16, 50\%, 69.2\% and $85.7 \%$ had no substitution at Par E. 25\%, 23.1\% and 14.3\% had Asp435Asn substitution. Only 25\% and $7.7 \%$ at MIC 4 and 8 had Glu407Lys substitution.

Table (5) Levofloxacin MICs and substitutions observed in GyrA and ParE in ciprofloxacin-resistant and intermediate S. pneumoniae isolates.

\begin{tabular}{|c|c|c|c|}
\hline Variable(MIC) & 4 & 8 & 16 \\
\hline \multicolumn{4}{|l|}{ GyrA } \\
\hline No substitution & $4(100 \%)$ & $7(53.8 \%)$ & $3(21.4 \%)$ \\
\hline Glu85Lys & 0 (0\%) & $1(7.7 \%)$ & $0(0 \%)$ \\
\hline Ser81Phe & 0 (0\%) & $4(30.8 \%)$ & $8(57.2 \%)$ \\
\hline Ser81Tyr & 0 (0\%) & $1(7.7 \%)$ & $3(21.4 \%)$ \\
\hline \multicolumn{4}{|l|}{ Par E } \\
\hline No substitution & $2(50 \%)$ & $9(69.2 \%)$ & $12(85.7 \%)$ \\
\hline Asp435Asn & $1(25 \%)$ & $3(23.1 \%)$ & $2(14.3 \%)$ \\
\hline Glu407Lys & $1(25 \%)$ & $1(7.7 \%)$ & $\mathbf{0}(0 \%)$ \\
\hline
\end{tabular}

As shown in table 6, at Gatfloxacin MIC 2, 4, 8 and 16, 75\%, 66.6\%, 41.7\% and $11.1 \%$ had no substitution in Gyr A, only $11.1 \%$ of gatfloxacin resistant and intermediate isolates had Glu85Lys substitution at MIC 16. Also at MIC 2, 4, 8 and 16, there were 25\%, 16.7\%, 50\% and $44.5 \%$ Ser81Phe substitution respectively. At

MIC 4, 8 and 16 there were $16.7 \%, 8.3 \%$ and $33.3 \%$ had Ser81Tyr substitution.

Regarding Par E, at Gatfloxacin MIC 2, 4, 8 and 16, 75\%, 83.3\%, 66.7\% and

77.8\% had no substitution at Par E. 16.7\%, 33.3\% and 11.1\% had Asp435Asn

substitution at MIC 4, 8 and 16. Only $25 \%$ and $11.1 \%$ at MIC 2 and 16 had Glu407Lyssubstitution.

Table (6) Gatfloxacin MICs and substitutions observed in GyrA and ParE in ciprofloxacinresistant and intermediate $\mathrm{S}$. pneumoniae isolates

\begin{tabular}{|c|c|c|c|c|}
\hline Variable(MIC) & 2 & 4 & 8 & 16 \\
\hline \multicolumn{5}{|l|}{ GyrA } \\
\hline No substitution & $3(75 \%)$ & $4(66.6 \%)$ & $5(41.7 \%)$ & $1(11.1 \%)$ \\
\hline Glu85Lys & $0(0 \%)$ & $0(0 \%)$ & $0(0 \%)$ & $1(11.1 \%)$ \\
\hline Ser81Phe & $1(25 \%)$ & $1(16.7 \%)$ & $6(50 \%)$ & $4(44.5 \%)$ \\
\hline Ser81Tyr & $0(0 \%)$ & $1(16.7 \%)$ & $1(8.3 \%)$ & $3(33.3 \%)$ \\
\hline \multicolumn{5}{|l|}{$\operatorname{Par} \mathbf{E}$} \\
\hline \begin{tabular}{|l|} 
No substitution \\
\end{tabular} & $3(75 \%)$ & $5(83.3 \%)$ & $8(66.7 \%)$ & $7(77.8 \%)$ \\
\hline Asp435Asn & $\mathbf{0}(0 \%)$ & $1(16.7 \%)$ & $4(33.3 \%)$ & $1(11.1 \%)$ \\
\hline Glu407Lys & $1(25 \%)$ & $0(0 \%)$ & $0(0 \%)$ & $1(11.1 \%)$ \\
\hline
\end{tabular}

235 
As shown in table 7, at Moxifloxacin MIC 2, 4, 8 and 16, 50\%, 55.6\%, 40\% and 25\% had no substitution in Gyr A, only $11.1 \%$ of moxifloxacin resistant and intermediate isolates had Glu85Lys substitution at MIC 4. Also at MIC 2, 4, 8 and16, therewere $25 \%, 33.3 \%, 30 \%$ and62.5\%Ser81Phesubstitutionrespectively.AtMIC 2, 8 and 16 there were $25 \%, 30 \%$ and $12.5 \%$ had Ser81Tyr substitution.

Regarding Par E, at Moxifloxacin MIC 2, 4, 8 and 16, 75\%, 66.7\%, 80\%and $75 \%$ had no substitution at Par E. 25\%, 22.2\%, 10\% and 25\% had Asp435Asn substitution at MIC 2, 4, 8 and 16. Only $11.1 \%$ and $10 \%$ at MIC 4 and 8 had Glu407Lys substitution.

Table (7) Moxifloxacin MICs and substitutions observed in GyrA and ParE in ciprofloxacin-resistant and intermediate S. pneumoniae isolates

\begin{tabular}{|l|l|l|l|l|}
\hline Variable(MIC) & 2 & 4 & 8 & 16 \\
\hline GyrA & & & & \\
\hline No substitution & $2(50 \%)$ & $5(55.6 \%)$ & $4(40 \%)$ & $2(25 \%)$ \\
\hline Glu85Lys & $0(0 \%)$ & $1(11.1 \%)$ & $0(0 \%)$ & $0(0 \%)$ \\
\hline Ser81Phe & $1(25 \%)$ & $3(33.3 \%)$ & $3(30 \%)$ & $5(62.5 \%)$ \\
\hline Ser81Tyr & $1(25 \%)$ & $0(0 \%)$ & $3(30 \%)$ & $1(12.5 \%)$ \\
\hline Par E & & & & \\
\hline No substitution & $3(75 \%)$ & $6(66.7 \%)$ & $8(80 \%)$ & $6(75 \%)$ \\
\hline Asp435Asn & $1(25 \%)$ & $2(22.2 \%)$ & $1(10 \%)$ & $2(25 \%)$ \\
\hline Glu407Lys & $0(0 \%)$ & $1(11.1 \%)$ & $1(10 \%)$ & $0(0 \%)$ \\
\hline
\end{tabular}

\section{Discussion}

Mean age of our study group was $22.2 \pm 20.8$, and ranged from 1 to64, our study included 49 (62.8\%)males and 29 (37.2\%) females. Majority of ourstudygrouphadhighESR,WBCs and positive CRP. In study of Kargar et al. (11) $16(35.33 \%)$ were maleand $29(64.45 \%)$ were female.

Ninty one $(91 \%)$ of isolates in our study were resistant to ampicillin, $5.1 \%$ were intermediate and $3.8 \%$ were susceptible. Regarding cefaclor $83.3 \%$ were resistant, $7.7 \%$ were intermediate and $9 \%$ were susceptible. Erythromycin was resistant in $82.1 \%$ of isolates, intermediate in $10.3 \%$, and susceptible in $7.7 \%$. Regarding imipenem $10.3 \%$ of isolates were resistant. Tetracycline was resistant in $71.8 \%$ of isolates. Clarithromycin was resistant in only $6.4 \%$, also $10.3 \%$ of our isolates were resistant to the antibiotics

ceftriaxone.Trimethoprim/sulfamethox azoleresistant in $9 \%$ of our isolates.
The MICs of Ciprofloxacin, Levofloxacin, Gatifloxacin and Moxifloxacin were measured in this study and we found that, $44.9 \%$ of S. pneumonia isolates were resistant to ciprofloxacin, $11.5 \%$ were intermediate and $43.6 \%$ were sensitive. Regarding levofloxacin $42.3 \%$ of isolates were resistant, 9\% were intermediate, and $48.7 \%$ were sensitive. Over forty six $(46.1 \%)$ of our isolates were resistant to Gatfloxacin, $10.3 \%$ were intermediate, and $43.6 \%$ were sensitive. Regarding Moxifloxacin $46.2 \%$ of our isolates were resistant, $7.6 \%$ were intermediate, and $46.2 \%$ were sensitive. Also in study of Kargar et al. (11) the resistance percentages of all strains to tested antibiotics were as follows: ciprofloxacin $73.33 \%$,

Ofloxacin 53.33\%, Norfloxacin $48.89 \%$, and levofloxacin $42.22 \%$. The highest resistance was observed in patients in the age group of 31-40 years ${ }^{(11)}$. 
Regarding genetic substitution, we found that at ciprofloxacin MIC 2, 4, 8

and $16,75 \%, 55.6 \%, 33.3 \%$ and $30 \%$

had no substitution in Gyr A, only $10 \%$ of ciprofloxacin resistant and intermediate

isolates had Glu85Lys substitution at MIC 16. Also at MIC 2, 4,8and16,therewere $25 \%, 33.1 \%$,

$66.7 \%$ and $30 \%$ Ser81Phe substitution respectively. At MIC 4 and 16 there were $11.1 \%$ and $30 \%$ had Ser81Tyr substitution. On the other hand, at Par E, at ciprofloxacin MIC 2, 4, 8 and 16, $50 \%, 66.7 \%, 77.85$ and $10 \%$ hadno substitution at Par E. 25\%,22.2\%, $22.2 \%$ and $10 \%$ had Asp435Asn substitution. Only $25 \%$ and $11.1 \%$ at MIC 1 and 4 had Glu407Lys substitution.

Similar to our results, in studies of Bast et al. (12), Broskey et al. (13), Brueggemann et al. (14) the GyrA substitutions observed most often were at positions Ser81 (Phe or Tyr) (54\% of ciprofloxacin-resistant isolates) and Glu85 (Gly or Lys) (10\% of ciprofloxacin-resistant

olates). Overall, the most common genotype observed was Ser79Phe (ParC) and Ser81Phe (GyrA) (35\% of ciprofloxacinresistant isolates). Also in study of Korzheva et al. (15) substitutions at Ser81 in GyrA are believed to be the most commonly observed substitutions as these positions interact with the fluoroquinolone in the ternary complex.

Regarding levofloxacin, we found that at levofloxacin MIC 4, 8 and 16, $100 \%, 53.8 \%$ and $21.4 \%$ had no substitution in Gyr A, only $7.7 \%$ of levofloxacin resistant and intermediate isolates had Glu85Lys substitution at MIC8.AlsoatMIC8and16,there were $30.8 \%$ and $57.2 \%$ Ser81Phe substitution respectively. At MIC 8 and 16 there were $7.7 \%$ and $21.4 \%$ had Ser81Tyr substitution. In Par E, at levofloxacin MIC 4, 8 and 16,50\%, $69.2 \%$ and $85.7 \%$ had nosubstitution at Par E. 25\%, 23.1\% and $14.3 \%$ had Asp435Asn substitution. Only 25\% and $7.7 \%$ at MIC 4 and 8 had Glu407Lys substitution. On the other hand, previous studies reported that between $59 \%$ and $71 \%$ of isolates with levofloxacin MICs of $2 \mathrm{pg} / \mathrm{mL}$ had QRDR substitutions in $\operatorname{GyrA}^{(16,17)}$. Few studies have evaluated isolates with levofloxacin MICs of $1 \mathrm{pg} / \mathrm{mL}$. The MIC 90 of levofloxacin for $\mathrm{S}$. pneumoniae is $1 \mathrm{pg} / \mathrm{mL}$. The use of this MIC provides the greatest number of isolates for study and is a sensitive measure of shifts over time ${ }^{(8)}$.

Regarding gatfloxacin, we found thatatgatfloxacinMIC2,4,8and16, 75\%, $66.6 \%, 41.7 \%$ and $11.1 \%$ had no substitution in Gyr A, only $11.1 \%$ of gatfloxacin resistant and intermediate isolates had Glu85Lys substitution at MIC16.AlsoatMIC2,4,8and16, there were $25 \%, 16.7 \%, 50 \%$ and $44.5 \%$ Ser81Phe substitution respectively.AtMIC4,8and16there were $16.7 \%, 8.3 \%$ and $33.3 \%$ had Ser81Tyr substitution. In Par E, at gatfloxacinMIC2,4,8and16,75\%, $83.3 \%, 66.7 \%$ and $77.8 \%$ had no substitution at Par E. 16.7\%, 33.3\% and $11.1 \%$ had Asp435Asn substitution at MIC 4, 8 and 16. Only 25\% and $11.1 \%$ at MIC 2 and 16 had Glu407Lyssubstitution.

Regarding moxifloxacin, we found thatatmoxifloxacinMIC2,4,8 and 16 , $50 \%, 55.6 \%, 40 \%$ and $25 \%$ had no substitution in Gyr A, only $11.1 \%$ of moxifloxacin resistantand intermediate isolates had Glu85Lys substitution at MIC 4. Also at MIC 2, 4,8and16,therewere $25 \%, 33.3 \%, \quad 30 \%$ and $62.5 \%$ Ser81Phe substitution 
respectively.AtMIC2,8and16 there were $25 \%, 30 \%$ and $12.5 \%$ had Ser81Tyr substitution. In Par E, at moxifloxacin MIC 2, 4, 8 and 16,75\%, $66.7 \%, \quad 80 \%$ and $75 \%$ hadno substitution at $\operatorname{Par}$ E. 25\%,22.2\%, $10 \%$ and $25 \%$ had Asp435Asn substitution at MIC 2, 4, 8 and 16. Only $11.1 \%$ and $10 \%$ at MIC 4 and 8 had Glu407Lys substitution.

In study of Kargar et al. (11) investigated the prevalence of mutations in the parE and gyrA genes and their role in the development of quinolone resistance. Their findings imply that only mutations in gyrA gene were resistant to ciprofloxacin,

susceptible to ofloxacin, and semisusceptible to levofloxacin. However, the isolates that had simultaneous mutations in both genes were completely resistant to ofloxacin and levofloxacin (18). Also, there are various opinions about the parE gene and its role in the development of resistance to quinolones; according to research by Kawamura-Sato et al. (19) in Japan and Credito et al. ${ }^{(20)}$ in the United States, isolates that had parE gene mutations, along with mutations in the gyrA gene, had higher resistance to ciprofloxacin, ofloxacin, norfloxacin, and lorfloxacin than mutants that did not have mutations in the parE gene. Findings of Kargar etal. (11) iscontrarytotheresultsofIpetal.

(21), which showed that the strains possess mutations in the parE gene are susceptible to quinolones and have no difference from the wild strains. In contrast to results of Ip et al. (21), for the first time in Iran, Kargar et al. (11) also illustrated that there is a significant correlation between mutations in the parE gene and resistance to norfloxacin, as strains that had mutations in the gyrA gene along with mutations in the parE gene showed higher levels of resistance to thisantibiotic.

Sierra et al. ${ }^{(22)}$ recently correlated mutagenic potency of the fluoroquinolones to likelihood of mutant selection. They found levofloxacin and moxifloxacin to be less mutagenic than ciprofloxacin and gemifloxacin and resistant mutants to be selected most commonly by ciprofloxacin followed by gemifloxacin, moxifloxacin and levofloxacin (22). In study of Brino et al. (23) most isolates had mutations at conventional sites in gyrA (codons for S81 and E85), the amino acid position most frequently reported to be associated with resistance of pneumococci to this class of agents. In addition, 3 of these 16 isolates had multiple mutations that included sites in parE. Fass et al. (24) found that only the MICs of levofloxacin and ofloxacin were increased with the introduction of this mutation intoparE.

Furtherore, no single mutants of gyrA were found in the isolates included in the study of Fukuda and Hiramatsu. (25) and single mutants of gyrA have been detected among strains selected in vitro onnewer

\section{Conclusion}

There is a strong relationship between these mutations and decrese susceptibility to the most fameous FQs to some extent,

\section{Recommandations}

We recommend:

1. Close attention to monitor fluoroquinolone susceptibility patterns and the association of multidrug resistance with fluoroquinolone resistance in isolates ofS.pneumoniae.

2. The increased prescription of fluoroquinolones as first-line therapy for common infections such as respiratory tract infection will facilitate the emergence of resistance to this class of compounds and promote the 
emergence of multidrug-resistant strains and, therefore, should be discouraged as it will undermine the efficacy of fluoroquinolones to treat more-seriousinfections.

3. Continued surveillance of respiratory tract isolates and other pathogens is important, and appropriate clinical use of fluoroquinolones is imperative as they become more widelyprescribed.

4. Further studies in larger numbers of patients are necessary to establish the role of genesubstitution

\section{References}

1. Mandell LA, Wunderink RG, Anzueto A, Bartlett JG, Campbell GD, Dean $\mathrm{NC}$, et al. Infectious Diseases Society of America/American Thoracic

fluoroquinolones, such as sparfloxacin or gatifloxacin $(\mathbf{2 5}, \mathbf{2 6})$, but are rarely reported for clinical isolates. This may reflect the relatively recent use of newer fluoroquinolones that select for gyrA mutations first. Also, isolates with a single mutation in gyrA may be overlooked if the MIC of the fluoroquinolone agent(s) used to screen for resistance is unchanged or only modestlyincreased. although this varies between strains and for eachdrug. in (QRDRs) in S. pneumonia isolates and resistance toFluoroquinlones.

The maintenance of such surveillance is valuable in the preparation of future therapy guidelines and could lead to new therapeutic strategies for FQresistant S.Pneumoniae.

Society consensus guidelines on the management of community-acquired pneumonia in adults. Clinical infectious diseases: anofficialpublication of the Infectious Diseases Society of America. 2007;44 Suppl 2:S27-72.

2. Patel SN, McGeer A,Melano R, Tyrrell GJ, Green K, Pillai DR, et al. Susceptibility of Streptococcus pneumoniae to fluoroquinolones in
Canada. Antimicrobial agents and chemotherapy.2011;55(8):3703-8.

3. Morrissey I, Farrell DJ, Bakker S, Buckridge S, Felmingham D. Molecular characterization and antimicrobial susceptibility of fluoroquinolone-resistant or susceptible Streptococcus pneumoniae from Hong Kong. Antimicrobial agents and chemotherapy.2003;47(4):1433-5.

4. de la Campa AG, Balsalobre L, Ardanuy C, Fenoll A, Perez-Trallero E, Linares $\mathrm{J}$, et al. Fluoroquinolone resistance in penicillin-resistant Streptococcus pneumoniae clones, Spain. Emerging infectious diseases. 2004;10(10):1751-9.

5. Fisher LM, Gould KA, Pan XS, Patel $\mathrm{S}$, Heaton VJ. Analysis of dual active fluoroquinolones in Streptococcus pneumoniae. The Journal of antimicrobial chemotherapy. 2003;52(2):312-3; author reply3-4.

6. Andersson MI, MacGowan AP. Development of the quinolones. The Journal of antimicrobial chemotherapy. 2003;51 Suppl 1:1-11.

7. Collins MD, Hutson RA, Hoyles L, Falsen E, Nikolaitchouk N, Foster G. Streptococcus ovis sp. nov., isolated from sheep. International journal of systematic and evolutionary microbiology. 2001;51(Pt3):1147-50.

8. Zhanel GG, Palatnick L, Nichol KA, Bellyou T, Low DE, Hoban DJ. Antimicrobial resistance in respiratory tract Streptococcus pneumoniae isolates: results of the Canadian Respiratory Organism Susceptibility Study, 1997 to 2002. Antimicrobial agents a chemotherapy. 2003;47(6):1867-74.

9. Tettelin H, Nelson KE, Paulsen IT, Eisen JA, Read TD, Peterson S, et al. Complete genome sequence of a virulent isolate of Streptococcus pneumoniae. Science. 2001;293(5529):498-506. 
10. Hoskins J, Alborn WE, Jr., Arnold J, Blaszczak LC, Burgett S, DeHoff BS, et al. Genome of the bacterium Streptococcus pneumoniae strain R6. Journal of bacteriology. 2001;183(19):5709-17.

11. Kargar M, MoeinJahromi F, Doosti A, Handali S. Molecular Investigation of Quinolone Resistance of Quinolone Resistance-Determining Region in Streptococcus pneumoniae Strains Isolated from Iran Using Polymerase Chain Reaction- Restriction Fragment Length Polymorphism Method. Osong public health and research perspectives. 2014;5(5):245-50.

12. Bast DJ, Low DE, Duncan CL, Kilburn L, Mandell LA, Davidson RJ, et al. Fluoroquinoloneresistance in clinical isolates of Streptococcus pneumoniae: contributions of type II topoisomerase mutations and efflux to levels of resistance. Antimicrobial agents

13. Broskey J, Coleman K, Gwynn MN, McCloskey L, Traini C, Voelker L, et al. Efflux and target mutations as quinolone resistance mechanisms in clinical isolates of Streptococcus pneumoniae. The Journal of antimicrobial chemotherapy. 2000;45 Suppl1:95-9.

14. Brueggemann AB, Coffman SL, Rhomberg $\mathrm{P}$, Huynh $\mathrm{H}$, Almer L, Nilius A, et al. Fluoroquinolone resistance in

Streptococcus pneumoniae in United States since 1994-1995. Antimicrobial agents and chemotherapy.2002;46(3):680-8.

15. Korzheva N, Davies TA, Goldschmidt R. Novel Ser79Leu and Ser81Ile substitutions in thequinolone

resistance-determining regions of ParC topoisomerase IV and GyrA DNA gyrasesubunits fromb recent fluoroquinolone-resistant Streptococcuspneumoniaeclinical isolates.Antimicrobial agents and chemotherapy.2005;49(6):2479-86.
16. Davies TA, Evangelista A, Pfleger S, Bush K, Sahm DF, Goldschmidt R. Prevalence of single mutations in topoisomerase type II genes among levofloxacin-susceptible clinical strains of Streptococcus pneumoniae isolated in the United States in 1992 to 1996 and 1999 to 2000. Antimicrobial agents and chemotherapy.2002;46(1):119-24.

17. Lim S, Bast D, McGeer A, de Azavedo J, Low DE. Antimicrobial susceptibility breakpoints and first-step parC mutations in Streptococcus pneumoniae: redefining

fluoroquinolone resistance. Emerging infectious diseases.2003;9(7):833-7.

18. Patel SN, Melano R, McGeer A, Green $\mathrm{K}$, Low DE. Characterization of the quinolone resistant determining regions in clinical isolates of pneumococci collected in Canada. Annals of clinical microbiology and

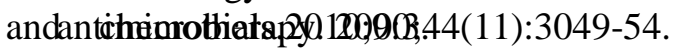

19. Kawamura-Sato K, Hasegawa T, Torii $\mathrm{K}$, Ito $\mathrm{H}$, Ohta $\mathrm{M}$. Prevalence of Ile$460-V a 1 / P a r E$ substitution in clinical Streptococcus pneumoniae isolates that were less susceptible fluoroquinolones.Current microbiology.2005;51(1):27-30.

Credito K, Kosowska-Shick K, McGhee P, Pankuch GA, Appelbaum PC. Comparative study of the mutant prevention concentrations of moxifloxacin, levofloxacin, and gemifloxacin against pneumococci. Antimicrobial agents and chemotherapy.2010;54(2):673-7.

20. Ip M, Chau SS, Chi F, Qi A, Lai RW. Rapid screening of fluoroquinolone resistance determinants in Streptococcus pneumoniae by PCR-restriction fragment length polymorphism and single- strandn conformational polymorphism. Journal of clinical microbiology.2006;44(3):970-5.

21. Sierra JM, Cabeza JG, Ruiz Chaler M, Montero T, Hernandez J, Mensa J, et al. The selection of resistance to and the mutagenicity of different 
fluoroquinolones in Staphylococcus aureus and Streptococcus

pneumoniae. Clinical microbiology and infection : the official publication of the European Society of Clinical Microbiology and Infectious Diseases.2005;11(9):750-8.

22. Brino L, Urzhumtsev A, Mousli M, Bronner C, Mitschler A, Oudet P, et al. Dimerization of Escherichia coli DNAgyrase B provides a structural mechanism for activating the ATPase catalytic center. The Journal of biological chemistry. 2000;275(13):9468-75.

23. Fass D, Bogden CE, Berger JM.
Quaternary changes in topoisomerase II may direct orthogonal movement of two DNA strands. Nature structural biology.1999;6(4):322-6.

24. Fukuda H, HiramatsuK. Primary targets of fluoroquinolones in Streptococcuspneumoniae.

Antimicrobialagents and chemotherapy.1999;43(2):41

25. Streptococcus pneumoniae by sparfloxacin: selective targeting of gyrase or topoisomerase IV by quinolones. Antimicrobial agents and chemotherapy. 1997;41(2):471-4. 